\title{
EDITORIAL
}

\section{The skeleton: no bones about it}

\author{
Colin Farquharson and Katherine Staines
}

Bone Biology Group, The Roslin Institute, Royal (Dick) School of Veterinary Studies, University of Edinburgh, Edinburgh EH25 9RG, UK

(Correspondence should be addressed to C Farquharson; Email: colin.farquharson@roslin.ed.ac.uk)

The skeleton is composed of bone and cartilage. These tissues are made of an exquisite assembly of functionally distinct cell populations and are required to support the structural, biochemical and mechanical integrity of the skeleton. The functions of the skeleton, which include its role in growth, locomotion, ion homeostasis and the protection of vital organs, are fundamental to the healthy organism. Development, growth and maintenance of the skeleton is under complex endocrine and genetic control and depends on a tight integration of cellular events within the skeleton and within the systems that deliver and accumulate mineral for hydroxyapatite formation (Karsenty 2003).

With few exceptions, bone formation is dependent on the development of the cartilage anlagen from mesenchymal precursors. These cartilage models of the future bones are replaced by bone through endochondral ossification, a process also responsible for linear bone growth at the epiphyseal plate during pre- and post-natal life (Kronenberg 2003). In essence, the laying down of a cartilage matrix by the chondrocyte, the primary cell type of cartilage, is a pre-requisite for future bone modelling and remodelling by the osteoblast and osteoclast. To fully appreciate the contribution of both cartilage and bone elements to skeletal physiology (and pathology), it is essential that we more thoroughly understand the molecular events responsible for the integration of the various signals and cues that impinge on, and direct, the activities of cartilage and bone cells.

To this goal, considerable advances have been made in our understanding of the regulatory mechanisms responsible for the formation and maintenance of the skeleton. This has been achieved through various classical in vitro and in vivo approaches including the analysis of specific knockout animals, transgenic mice and animals which bear spontaneous mutations. This progress in our understanding has been captured in these three thematic reviews, where the authors, in addition to providing a general review of endochondral ossification and bone formation and resorption, have focussed on some of the recent advances in chondrocyte, osteoblast and osteoclast biology.

In the first thematic review, Mackie et al. (2011) summarise the current concepts of growth plate chondrocyte activity and growth plate function. Chondrocyte proliferation is essential for bone growth, and the authors highlight recent advances in the control of chondrocyte proliferation by Indian hedgehog and bone morphogenetic proteins (BMPs). Of particular interest is the recently recognised cooperation between Schnurri proteins -2 and -3 and receptor-Smads in the regulation of BMP signalling (Jones et al. 2010). In addition, a role for the transcriptional repressor, TRPS1, which interacts with the activator form of Gli3, in chondrocyte cycle progression has been proposed (Wuelling et al. 2009). It is also worthy of mention that TRPS1 appears to be able to regulate chondrocyte hypertrophy by directly inhibiting PTHrP expression and RUNX2 function. This suggests that TRPS1 has an important function in the fine-tuning of cell-cycle exit and in the transition between the proliferative and the hypertrophic chondrocyte states (Napierala et al. 2008).

Mineralisation of the hypertrophic chondrocyte matrix is followed by vascular invasion of the growth plate, and the subsequent deposition of the bone matrix by osteoblasts on the vertical cartilage remnants remaining after osteoclastic matrix resorption. The regulation of invading cells is now considered to be an active process in which the chondrocytes produce factors that promote and attract the invasion of endothelial cells, osteoblasts and osteoclasts. In the second thematic review, new insights into osteoblast biology are summarised with a particular focus on the role of phosphatidylinositol 3-kinase (PI3K) signalling and its downstream effector, AKT (Guntur \& Rosen 2011). Of topical interest is the ability of insulin/IGF1 signalling to up-regulate PI3K activity and, via the Forkhead group of transcription factors (FOXOs), influence osteoblast function and energy metabolism through altered osteocalcin and OST-PTP expression (Rached et al. 2010, Kousteni 2011). This is in addition to the previously recognised effects of PI3K signalling on the osteoblast-specific transcription factors, RUNX2, Osterix and ATF4. Interestingly, recent data suggest that conditional loss of Pten, a phosphatase that inactivates $\mathrm{PI} 3 \mathrm{~K}$ signalling, in osteoprogenitors results in increased proliferation of osteoblast progenitors and stimulation of fibroblast growth factor signalling (Guntur et al. 2011). 
The last thematic review concentrates on the bone resorbing osteoclast whose function is synchronised to that of the osteoblast during bone remodelling to ensure bone mass homeostasis (Mellis et al. 2011). The focus of this thematic review is the key signalling pathways involved in osteoclast formation and function. The coupling of osteoblast and osteoclast function is accepted to involve the receptor activator of nuclear factor kappa- $\beta$ (RANK)/osteoprotegrin $(\mathrm{OPG}) / \mathrm{RANK}$ ligand (RANKL) system, and this review summarises in detail the downstream signalling events that occur after RANK receptor activation, which result in osteoclast formation and bone resorption. Other pathways are also involved in osteoclast formation and one of these involves the ratio of the two isoforms - liver-enriched inhibitory protein (LIP) and liver-enriched activator protein (LAP) - of the transcription factor CCAAT enhancer-binding protein $\beta$. The ratio of LIP and LAP, which is modulated by the mechanistic target of rapamycin signalling pathway, regulates osteoclastogenesis through MafB (Smink et al. 2009). Once formed, osteoclast attachment to the bone matrix is essential for resorption and this is mediated by various signalling pathways such as those initiated by SRC kinase. SRC phosphorylation of cortactin and gelsolin is important for actin polymerisation and podosome turnover whereas phosphorylation of spleen tyrosine kinase, SYK, promotes the activation of small GTPases and cytoskeletal reorganisation (Itzstein et al. 2011).

The aim of these thematic reviews is to bring together in one collection an appreciation of our current understanding of the key factors, e.g. endocrine, genetic and signalling molecules, that regulate skeletal function via interactions with cells of both cartilage and bone. Each individual cell type (including the osteocyte, the terminally differentiated osteoblast) does not live in isolation or in a vacuum and there is much crosstalk and communication between them and also with their collagenous-rich extracellular matrix. Together, these cellular activities and matrix interactions result in the formation of a complex organ that is indispensable for life.

\section{Declaration of interest}

The authors declare that there is no conflict of interest that could be perceived as prejudicing the impartiality of the research reported.

\section{Funding}

The authors acknowledge the Institute Strategic Programme Grant Funding from the BBSRC (C F) and BBSRC studentship funding (K S) for support.

\section{References}

Guntur AR \& Rosen CJ 2011 The skeleton: a multi-functional complex organ. New insights into osteoblasts and their role in bone formation: the central role of PI3kinase. Journal of Endocrinology 211 123-130. (doi:10. 1530/JOE-11-0175)

Guntur AR, Reinhold MI, Cuellar J \& Naski MC 2011 Conditional ablation of Pten in osteoprogenitors stimulates FGF signaling. Development 138 1433-1444. (doi:10.1242/dev.058016)

Itzstein C, Coxon FP \& Rogers MJ 2011 The regulation of osteoclast function and bone resorption by small GTPases. Small GTPases 2 117-130. (doi:10. 4161/sgtp.2.3.16453)

Jones DC, Schweitzer MN, Wein M, Sigrist K, Takagi T, Ishii S \& Glimcher LH 2010 Uncoupling of growth plate maturation and bone formation in mice lacking both Schnurri-2 and Schnurri-3. PNAS 107 8254-8258. (doi:10.1073/pnas.1003727107)

Karsenty G 2003 The complexities of skeletal biology. Nature 423 316-318. (doi:10.1038/nature01654)

Kousteni S 2011 FoxO1: a molecule for all seasons. Journal of Bone and Mineral Research 26 912-917. (doi:10.1002/jbmr.306)

Kronenberg HM 2003 Developmental regulation of the growth plate. Nature 423 332-336. (doi:10.1038/nature01657)

Mackie EJ, Tatarczuch L \& Mirams M 2011 The skeleton: a multi-functional complex organ. The growth plate chondrocyte and endochondral ossification. Journal of Endocrinology 211 109-121. (doi:10.1530/ JOE-11-0048)

Mellis DJ, Itzstein C, Helfrich MH \& Crockett JC 2011 The skeleton: a multifunctional complex organ. The role of key signalling pathways in osteoclast differentiation and in bone resorption. Journal of Endocrinology 211 131-143. (doi:10.1530/JOE-11-0212)

Napierala DK, Sam K, Morello R, Zheng Q, Munivez E, Shivdasani RA \& Lee B 2008 Uncoupling of chondrocyte differentiation and perichondrial mineralization underlies the skeletal dysplasia in tricho-rhino-phalangeal syndrome. Human Molecular Genetics 17 2244-2254. (doi:10.1093/hmg/ddn125)

Rached M-T, Kode A, Silva BC, Jung DY, Gray S, Ong H, Paik J-H, dePinho RA, Kim JK, Karsenty G et al. 2010 FoxO1 expression in osteoblasts regulates glucose homeostasis through regulation of osteocalcin in mice. Journal of Clinical Investigation 120 357-368. (doi:10.1172/JCI39901)

Smink JJ, Begay V, Schoenmaker T, Sterneck E, de Vries TJ \& Leutz A 2009 Transcription factor $\mathrm{C} / \mathrm{EBP}$ beta isoform ratio regulates osteoclastogenesis through MafB. EMBO Journal 28 1769-1781. (doi:10.1038/emboj.2009.127)

Wuelling M, Kaiser FJ, Buelens LA, Braunholz D, Shivdasani RA, Depping R \& Vortkamp A 2009 Trps1, a regulator of chondrocyte proliferation and differentiation, interacts with the activator form of Gli3. Developmental Biology 328 40-53. (doi:10.1016/j.ydbio.2009.01.012)

Received in final form 11 July 2011

Accepted 21 July 2011 